\section{$\underset{\substack{\text { hommes } \\ \text { \& migrations }}}{ }$}

\section{Hommes \& migrations}

Revue française de référence sur les dynamiques

migratoires

$1311 \mid 2015$

Femmes et migrations

\title{
Congrès de musique arabe du Caire
}

L'intégrale publiée par la BnF

\section{François Bensignor}

\section{(2) OpenEdition \\ 1 Journals}

\section{Édition électronique}

URL : http://journals.openedition.org/hommesmigrations/3378

DOI : 10.4000/hommesmigrations.3378

ISSN : 2262-3353

\section{Éditeur}

Musée national de l'histoire de l'immigration

\section{Édition imprimée}

Date de publication : 1 juillet 2015

Pagination : 174-179

ISBN : 978-2-919040-32-2

ISSN : $1142-852 X$

Référence électronique

François Bensignor, "Congrès de musique arabe du Caire », Hommes \& migrations [En ligne], 1311 | 2015, mis en ligne le 23 février 2016, consulté le 14 septembre 2020. URL : http://

journals.openedition.org/hommesmigrations/3378 


\title{
CONGRÈS DE MUSIQUE ARABE DU CAIRE L'INTÉGRALE PUBLIÉE PAR LA BNF
}

\author{
par FRANÇOIS BENSIGNOR
}

$\mathrm{L}^{\mathrm{c} \cos }$ e Congrès de musique arabe du Caire marque l'histoire de la musique. Organisé du 14 mars au 3 avril 1932 sous l'égide du roi Fouad ler d'Égypte par le baron Rodolphe d'Erlanger, il avait pour ambition d'embrasser les traditions de l'Égypte, du Maghreb et du Machrek. Des délégations de musiciens enregistrèrent des formes musicales anciennes, transmises depuis des générations, afin que soient étudiées leurs spécificités. Musicologues arabes et occidentaux débattirent en commissions, préparant l'avènement de la musique panarabe modernisée.

Les événements historiques et géopolitiques postérieurs au Congrès de musique arabe du Caire allaient interrompre les travaux d'intérêt culturel entrepris par ses participants. Et il fallut attendre la fin des années 1980 pour que les premiers chercheurs en musicologie commencent à exhumer les trésors des archives réunis en 1932, puis transmis à Berlin, Londres et Paris. La Bibliothèque nationale de France $(\mathrm{BnF})$, destinataire de l'ensemble des enregistrements réalisés lors du Congrès du Caire, en publie pour la première fois l'intégralité, sous la forme d'un coffret de $18 \mathrm{CD}$, accompagné d'un livret trilingue français, anglais et arabe de 256 pages.

"Le Congrès du Caire de 1932 est un mythe pour tous les chercheurs et amateurs de la musique arabe en particulier et de la musique en général. Raresétaient ceux qui avaient,jusqu'à cette édition, accès aux enregistrements de ce Congrès", écrit Chérif Khaznadar' dans sa préface. II faut saluer sa détermination à rendre possible cette toute première édition. Sans son engagement, la subtile complexité de l'art musical arabe révélée par ces enregistrements n'aurait peut-être pas été portée à la connaissance du monde globalisé. Déjà, dans les années 1990, à la demande du ministère de la Culture du Maroc, il rendait possible l'édition de l'ensemble des noubas transmises jusqu'à nos jours par la tradition andalouse marocaine al-aala en un coffret de 73 CD. La publication de l'intégrale du Congrès du Caire devait marquer l'inauguration du Centre pour les musiques traditionnelles du monde qu'il envisage d'installer à Abu Dabi. Ce centre est encore à l'état de projet, mais le partenariat qu'il a initié entre l'Autorité pour le tourisme et la culture de l'Émirat et la BnF aboutit à l'édition du coffret en 2015.

\section{Le contexte et les participants}

Le Congrès de musique arabe du Caire se déroule juste après la "nahda", la renaissance culturelle arabe (1903-1930). On parle encore alors de "musique orientale". Le Congrès ouvre une grande réflexionsurl'ensembledes musiquesquerecouvre ce terme, auquel se substitue celui de "musique arabe". "C'est l'acte de naissance de la musique 
Une partie des congressistes, photo extraite du livret du coffret, avec l'aimable autorisation de la BnF. (c)D.R.

arabe moderne, dit Jean Lambert². Les années 1930 sont marquées par le nationalisme arabe. Après l'effondrement de l'Empire ottoman, l'indépendance de la Turquie et la montée du sionisme, les Arabes veulent aussi leur patrie. Or le nationalisme égyptien de l'époque se présente plutôt comme panarabe. C'est donc naturellement que Le Caire est choisi par les musicologues orientalistes, emmenés par le baron d'Erlanger' et des musicologues égyptiens pour accueillir la conférence."

Le Congrès rassemble pour la première fois des maîtres orientaux et occidentaux. Parmi les Occidentaux figurent les compositeurs hongrois Béla Bartok, allemand Paul Hindemith, tchèque Aloïs
Haba, français Henri Rabaud. Le célèbre critique musical Émile Vuillermoz est également invité. Les chercheurs allemands Robert Lachmann, Curt Sachs et Erich Von Hornbostel représentent la "musicologie comparée", nouvelle discipline qui ne survivra pas au nazisme. L'orientaliste et historien de la musique britannique Henry George Farmer participe à l'événement. Les musicologues orientalistes français sont représentés notamment par le baron Carra de Vaux, le père Xavier Collangettes, adjoint du baron d'Erlanger - atteint d'une maladie qui l'emportera sept mois après son ouverture, celui-ci ne peut participer au congrès qu'il a organisé -, et Alexis Chottin, qui

2. Jean Lambert est membre du Centre de recherche en ethnomusicologie (CREM) du laboratoire d'ethnologie de l'université de Nanterre. Spécialiste des musiques du Yémen depuis plus de trente ans, il élargit son champ d'intérêt à l'histoire de la musique arabe au XXe siècle. II a assuré, auprès de Pascal Cordereix, la direction scientifique de la publication du coffret du Congrès de musique arabe du Caire.3. Orientaliste franco-britannique, Rodolphe d'Erlanger s'est illustré comme peintre, musicien et musicologue. Il quitte Paris pour s'installer en Tunisie, où il fait bâtir son palais, Ennajma Ezzahra (Planète Vénus), sur le golfe de Tunis. II initie et coécrit La musique arabe, ouvrage en 6 volumes, qui reste une référence. Considérant sa grande connaissance en ce domaine, le roi Fouad ler d'Égypte lui confie l'organisation du Congrès de musique arabe du Caire. 


\section{MUSIQUES}

dirige alors le Conservatoire de musique arabe de Rabat au Maroc.

"Côté arabe, la musicologie est en pleine émergence, explique Jean Lambert. Excepté l'Égyptien Mahmud al-Hifnî, auteur d'une thèse avec l'un des musicologues allemands, il s'agit plutôt d'amateurs éclairés ou de lettrés, mais aussi de musiciens. Citons notamment Alî al-Darwish, venu d'Alep ; Wadî Sabrâ, le directeur du conservatoire de Beyrouth au Liban; le maitre turc Mehmed Rauf Yekta bey, auteur d'un grand article sur la musique de Turquie dans l'Encyclopédie de la musique de Lavignac. La délégation égyptienne est importante, sûre d'elle-même et animée d'idées nationalistes. Parmi elle, Mustafâ Ridâ bey, Safar Ali bey et un jeune compositeur à l'avenir prometteur, Muhammad Abd alWahhâb (Mohammed Abdel Wahab)."

\section{Les enjeux du Congrès}

Le Congrès se déroule sur trois semaines. Avant les réunions plénières qui ont lieu du 28 mars au 3 avril 1932, des commissions spécialisées se réunissent à huis clos entre le 14 et le 27 mars. Certaines d'entre elles sont le théâtre de débats assez animés, portant sur les échelles et les formes musicales, les instruments, la pédagogie, l'histoire de la musique. Pour les Égyptiens, il s'agit de définir les normes musicales arabes, afin que la musique arabe puisse être considérée sur des bases comparables à celles de la musique occidentale. La transcription est une des préoccupations fondamentales du congrès. Celle des intervalles constitue la principale difficulté, l'écriture impliquant une réduction normative.

"Les théoriciens égyptiens étaient parvenus à la conclusion que l'on pouvait diviser l'échelle musicale arabe en 24 quarts de ton, l'échelle occi-

Le joueur de oud égyptien Safar Ali,

photo extraite du livret du coffret, avec l'aimable autorisation de la BnF. (c).R.

dentale étant divisée en 12 demi-tons, dit Jean Lambert. Mais les pratiques musicales ne correspondaient pas à cette théorie. Dans la musique syrienne, par exemple, on trouve des trois quarts de ton qui sont presque des tons, d'autres qui sont un peu plus bas et se rapprochent du demi-ton. D'autres théories, notamment celles du lima et du coma, qui sont des micro-intervalles, permettraient de mesurer ces intervalles. Mais leurs représentants ne participant pas au congrès, la discussion entre ces deux écoles n'a pas pu avoir lieu. Le musicologue turc a bien opposé quelques réticences, la musique turque n'étant pas normalisée en demi-tons. Le Syrien Alî al-Darwich n'était pas non plus d'accord, mais du fait qu'il était rémunéré par les Égyptiens, défendre son point de vue lui devenait plus difficile. Malgré les mesures effectuées, qui contredisaient leur théorie, les musicologues égyptiens n'en ont fait qu'à leur tête en décidant de moderniser selon leurs critères. À l'issue du Congrès, l'Institut de musique orientale du Caire s'est transformé en Institut de musique arabe du 
Caire et cette vision moderniste s'est imposée à la nouvelle génération."

\section{Le contenu musical}

L'Égypte est largement représentée par ses délégations de musiciens. Dix disques du coffret sont consacrés à leurs enregistrements, dont trois aux "muwashshah". Comme le précise le livret: "Les 'muwashshah' sont des poèmes multirimes et multimètres en arabe classique et en arabe médian. Ils sont généralement chantés en chœur, laissant seulement de courtes parties à un soliste, et sur des cycles rythmiques souvent complexes, comme 10 temps, 13 temps, 14 temps, 32 temps, etc." Cette forme est héritée de la poésie araboandalouse, essentiellement amoureuse et chantée, sur laquelle s'exerça le génie de Ziryabª

Jean Lambert explique : "La forme muwashshah s'est transférée du Maghreb en Égypte, vers les XI et XIle siècles. Dès le Moyen Âge, une tradition proprement syro-égyptienne de muwashshah s'est développée. Les pièces les plus anciennes chantées en Syrie datent de cette époque. Étant le pur fruit de l'oralité, on n'en connait pas les auteurs. Elles ont été rapatriées au XIXe siècle en Égypte. Le royaume égyptien, florissant sur le plan économique à l'époque, attirait les talents syro-libanais de toutes disciplines, dont les musiciens et les gens de théatre. C'est probablement la raison pour laquelle les Syriens étaient absents du congrès : les Syro-Libanais présents étaient considérés comme des Égyptiens."

D'autres formes de musiques égyptiennes profanes, savantes ou populaires, et religieuses figurent aussi parmi les enregistrements. Particulièrement impressionnants sont les chants des
Bédouins du Fayyûm (Fayoum), rassemblés sur un $C D$; les rituels soufis, tout particulièrement les chants de danses de possession consacrées au zâr ; les chants et prières de l'Église copte.

Le Maghreb est assez bien représenté. Les écoles arabo-andalouses de Tlemcen en Algérie et de Fès au Maroc se partagent trois CD. "La délégation marocaine était dirigée par la personnalité importante de Muhammad Qaddûr Ben Ghabrit, ministre plénipotentiaire du Maroc (alors sous mandat français) en France et recteur de la Mosquée de Paris, explique Jean Lambert. Lui-même, originaire de Tlemcen, avait quitté l'Algérie pour éviter la conscription française lors du premier conflit mondial. Devenu l'un des bras droits du roi Mohamed V, c'est une représentation nationale qu'il dirige au Congrès du Caire." Quant au malouf de Tunisie, il est mis en valeur sur deux albums. Jean Lambert: "L'ensemble tunisien avait été spécialement formé par Erlanger et le musicologue syrien Alî al-Darwich pour présenter l'état de la tradition tunisienne, telle qu'elle va quasiment disparaitre par la suite."

Trois CD sont consacrés aux musiques du Machrek. Le maqam d'Irak y tient une place de choix, celle accordée aux musiques de Syrie et de Turquie étant assez réduite. "On peut regretter que la Syrie ne figure que sur deux plages d'un disque, alors qu'il y avait à l'époque de grands chanteurs et musiciens syriens", commente Jean Lambert.

\section{Les enregistrements}

Le baron d'Erlanger comme les musicologues allemands entendaient profiter du rassemblement de musiciens arabes invités au congrès pour les enregistrer. Les organisateurs égyptiens, 


\section{MUSIQUES}

qui ne maîtrisaient pas encore cette technique, ne l'avaient pas prévu. Les techniciens français contactés par Erlanger ayant reculé devant la difficulté, c'est la compagnie britannique Gramophone, future His Masters' Voice (La Voix de son maître), qui relève le défi.

"Les enregistrements sont réalisés en studio à des fins d'exploitation musicologique scientifique, précise Jean Lambert. Sur presque chaque plage est joué un diapason afin que l'on puisse repérer la hauteur de la mélodie. La plupart des orchestres enregistrés sont conçus pour être des référents de la tradition musicale qu'ils représentent. D'une certaine manière, ils produisent une musique qui est déjà presque obsolète en 1932."

À travers l'important travail mené sur ces enregistrements conservés à la BnF, Bernard Moussalis dénombre 360 enregistrements effectués, dont certains en double, le Recueil des travaux du Congrès énumérant 171 disques $25 \mathrm{~cm}$ ou $30 \mathrm{~cm}$. Durant leur transport d'Alexandrie à Manchester, 16 matrices ont été cassées. L'intégrale des enregistrements publiés dans le coffret recouvre 334 plages. Les enregistrements ont été faits en gravure directe sur disques 78 tours avec la technologie électrique, toute nouvelle à l'époque, qui donne au son une bien meilleure dynamique par rapport à la technologie physique utilisée jusqu'alors.

"À la demande des interprètes, il n'y a pas eu d'édition commerciale des enregistrements réalisées par la Gramophone, explique Pascal Cordereix 6 . Quatre collections officielles ont été tirées des enregistrements : une est à la British Library, une autre au Phonogrammarchiv de Berlin, une au Musée Guimet à Paris et une à la BnF. Le Congrès du Caire est une de nos collections sonores emblématiques, par sa rareté et par la valeur de ses enregistrements. Nous sommes très sensibles à l'histoire de l'inter- prétation à travers le XXe siècle, à la façon dont l'enregistrement a pu en garder trace, tout particulièrement dans le domaine des musiques traditionnelles extra-occidentales. L'histoire de l'édition phonographique nous intéresse aussi. Notre intérêt va à cette double approche culturelle de l'histoire de l'interprétation et de l'histoire de l'industrie du disque."

\section{L'édition}

En 1988, à l'initiative de Marie-France Callas, alors directrice du département de la phonothèque et de l'audiovisuel à la Bibliothèque nationale, une édition partielle du Congrès du Caire avait été publiée, en coédition avec l'Institut du monde arabe (IMA) qui venait d'être inauguré, dans un coffret de trois CD. "Les musicologues Christian Poché et Bernard Moussali en avaient assuré la partie scientifique, poursuit Pascal Cordereix. Bernard Moussali préparait en parallèle sa thèse sur le Congrès du Caire, qu'il n'a malheureusement pas pu soutenir, étant décédé en 1996. Jean Lambert, étant son légataire testamentaire, a pu repartir des matériaux réunis par Bernard Moussali afin de rédiger le livret de l'édition intégrale."

La collection sonore du Congrès du Caire est l'une des premières à avoir été numérisée en "copie droite", sans rien toucher au son, par la BnF à partir de 1996. Depuis, la technologie de restitution sonore a beaucoup progressé. Le superbe travail de restauration réalisé permet ainsi d'apprécier la qualité des œuvres dans une dynamique sonore jusqu'alors inédite. "Le plus gros défi était d'éliminer les ronflettes liées aux procédés d'enregistrement de l'époque, ainsi que les bruits parasites - type voitures qui passent, bruits de portes, etc. - tout en conservant la couleur, le signal utile

\footnotetext{
5. Bernard Moussali (1953-1996) avait dédié sa trop courte carrière à la littérature et à la musique arabe. Professeur à la Sorbonne, il avait choisi le Congrès de musique arabe du Caire pour sujet de la thèse de doctorat qu'il n'a pu achever.

6. Pascal Cordereix, directeur du département de l'audiovisuel à la Bibliothèque nationale de France, a dirigé l'édition du coffret du Congrès de musique arabe du Caire avec Jean Lambert.
} 
des instruments, et notamment les basses des percussions, explique Pascal Cordereix. La grande réussite de ce travail est d'avoir préservé l'originalité des enregistrements faits par les ingénieurs de la Gramophone - sans altérer le signal, sans utiliser la compression dynamique, ni même l'équalisation - en supprimant les distorsions et autres bruits." Le travail de restitution effectué par Luc Verrier, expert en numérisation et préservation numérique au département audiovisuel de la $B n F$, et son équipe est de ce point de vue assez extraordinaire.

\section{L'importance des archives}

L'imposant livret publié dans le coffret est, pour l'amateur comme pour le chercheur, une précieuse source de renseignements sur les répertoires, les artistes et les pièces enregistrées. "Je tiens à rendre hommage à Bernard Moussali, dont le travail formidable était inconnu du public, en dehors du premier coffret publié par l'IMA, dit Jean Lambert. II avait notamment identifié toutes les pièces par les textes chantés, les modes, les cycles rythmiques, les fonctions des musiques rituelles. II avait largement expliqué leurs aspects historiques et religieux." S'il regrette d'avoir dû, en raison des contraintes d'édition du livret, synthétiser le contenu des fiches réalisées par Bernard Moussali, Jean Lambert annonce leur future publication intégrale sur Gallica, le site web de la BnF. Pascal Cordereix confirme également la volonté de la BnF de rendre accessible en ligne l'ensemble des enregistrements. "Il est urgent de s'occuper des archives de la musique arabe, qui sont fortement menacées, insiste Jean Lambert. En Irak, une partie des archives qui avaient été constituées par Shéhérazade Hassan dans les années 1970 ont brûlé. Ce qui reste est préservé à Bagdad, mais on ne sait pas dans quelles conditions. Les Égyptiens ne s'intéressent pas aux archives de leur musique. Heureusement, la Fondation Amar a récupéré la collection de 6000 disques 78 tours égyptiens, qu'elle s'emploie à numériser et à publier. Au Liban, les choses sont fragiles. En Syrie, aucune institution n'existait pour s'occuper de la musique et on ne sait rien de ce qui se passe de ce point de vue. Nos amis musiciens sont, pour la plupart d'entre eux, réfugiés au Liban, en France ou dans d'autres pays. Le Yémen est sous les bombes... C'est dramatique... II faudrait que de jeunes Arabes s'intéressent à leur patrimoine musical. S'ils perdent cette mémoire, que leur restera-t-il ?"

"Les Tunisiens font des efforts dans ce sens. Ennejma Ezzahra, l'ancien palais du baron d'Erlanger à Sidi Bou Saïd (Tunis), est devenu le Centre des musiques arabes et méditerranéennes. Un partenariat de travail a été établi avec le Centre de recherche en ethnomusicologie (CREM) de l'université de Nanterre ${ }^{8}$. Nous devons être conscients $d u$ fait que pour conserver cette mémoire, il faut mettre les techniques du numérique les plus avancées au service du patrimoine le plus authentique." I

/// Congrès de musique arabe du Caire, coffret de 18 CD, Paris, éd. de la BnF, 2015, 199 euros. 